\title{
FDK-Type Reconstruction Algorithms For The Reverse Helical Trajectory
}

\author{
Z. Yu, Student Member, IEEE, F. Noo, Member, IEEE, F. Dennerlein, G. Lauritsch, and J. Hornegger
}

\begin{abstract}
For emergency cases in the interventional room, 3D long-object cone-beam $(\mathrm{CB})$ imaging using a $\mathrm{C}$-arm system could save valuable time and reduce risks to the patient by avoiding the traditionally-used CT scan, and thus could potentially be a crucial tool for patient health. To accomplish such a task, the reverse helix is an attractive trajectory, however theoreticallyexact and stable(TES) reconstruction with a reverse helix is challenging. Two TES solutions are available, but both of them come with a heavy computational load and some issues in terms of image quality. This work proposes three new approximate reconstruction algorithms for the reverse helix that are stable and efficient, and thus practical. Though not exact, reconstruction results obtained from all three methods appear acceptable.
\end{abstract}

\section{INTRODUCTION}

For emergency cases in the interventional room, 3D longobject cone-beam $(\mathrm{CB})$ imaging using a $\mathrm{C}$-arm system (e.g., to assess status of the aorta or the spine, or to perform wholebody angiography) could save valuable time and reduce risks to the patient by avoiding transfer to another room for a CT scan. Thus, it could be a vital tool for patient care. However, performing such an imaging task is difficult for a $\mathrm{C}$-arm system in terms of scanning geometry, because significant constraints control the mechanical motions and there is no slip-ring technology for C-arm systems. In this context, the reverse helix suggested in [1] is an attractive solution.

For theoretically-exact and stable (TES) reconstruction, the reverse helix is very challenging due to poor R-line coverage within the field of view; there is a large region, called the core, of points of interests that do not belong to R-lines. An $\mathrm{R}$-line is a line that connects two points on a continuous curve. The group of Utah [2] has found a solution by applying the DBP-HT method in the R-line region and employing an FBP formulation of the method of Grangeat in the core region. Another solution was proposed by the group of Chicago [3], which utilizes the same DBP-HT formula for the R-line region

Manuscript received May 10, 2011. This work was partially supported by a grant from Siemens AG, Healthcare Sector and by the U.S. National Institutes of Health (NIH) under Grant No. R21 EB009168. The concepts presented in this paper are based on research and are not commercially available. Its contents are solely the responsibility of the authors and do not necessarily represent the official views of the NIH.

Zhicong $\mathrm{Yu}$ is with the Department of Radiology, the University of Utah, Salt Lake City, USA (e-mail: zyu@ucair.med.utah.edu). He is also a Ph.D candidate with the Chair of Pattern Recognition, Friedrich-AlexanderUniversity of Erlangen-Nuremberg, Erlangen, Germany.

Frederic Noo is with the Department of Radiology, the University of Utah, Salt Lake City, USA (e-mail: noo@ucair.med.utah.edu).

Frank Dennerlein and Guenter Lauritsch are with Siemens AG, Healthcare Sector, Forchheim, Germany.

Joachim Hornegger is with the Chair of Pattern Recognition, FriedrichAlexander-University of Erlangen-Nuremberg, Erlangen, Germany. and the Pack-Noo formula [4] for the core. Both methods produce TES results while allowing axial data truncation. However, both of them require one forward projection and two backprojections, and thus are not computationally efficient. Moreover, they come with some issues in terms of image quality: the reconstructions over the set of points that belong and do not belong to R-lines are not easily compatible in terms of resolution, and this can yield undesirable artifacts.

In this work, we propose three new FDK-type reconstruction methods for the reverse helical trajectory. The first method, called Fusion-RFDK (R stands for ramp) employs a horizontal ramp kernel based filtering, whereas the second method called Fusion-HFDK ( $\mathrm{H}$ stands for Hilbert) utilizes a horizontal Hilbert transform along with differentiation between successive projections along the scan. The motivation for FusionHFDK over Fusion-RFDK comes from studies related to the conventional helical trajectory [5], [6], which have shown that oblique filtering, along the direction of the tangent to the helix, can significantly reduce CB artifacts; the differentiation step in Fusion-HFDK induces an oblique component within the filtering operation. To understand how the third method was conceived, note that TES or near-TES reconstruction can be easily achieved within the regions covered by $\pi$-lines; a $\pi$ line is a line segment that connects any two points on a helix that are separated by less than $360^{\circ}$. The third method, called Voxel-Dependent-HFDK, make proficient use of this feature, so that it nearly produces TES reconstructions within the $\pi$ line regions.

All three proposed methods require only one backprojection and involve no forward projection, and thus are computational efficient. In addition, since all three methods are of FDK-type, they are simple and stable, and they are easily implemented on current $\mathrm{C}$-arm systems using existing hardware. Computersimulation results show no artifacts related to the transition from the core to the region outside the core. Moreover, our preliminary evaluations of CB artifacts show that all three methods produce images of acceptable quality. Hereafter, we first explain the scan geometry, then describe all three methods and show our evaluation results.

\section{GEOMETRY AND NOTATION}

We denote the spatial distribution of the linear attenuation coefficient as $f(\underline{x})$, where the point $\underline{x}$ has $(x, y, z)$ as its Cartesian coordinates. The function $f(\underline{x})$ is assumed to be smooth and compactly supported within a cylinder $\Omega$ of radius $r$, which is centered along the $z$-axis, as shown in Figure 1. Here $\Omega$ is usually referred to as the region of interest (ROI). 


\section{A. The reverse helix}

The reverse helical source trajectory is based on two motions, one is a circular rotation around the $z$-axis, and the other is a linear unidirectional movement along the $z$-axis. Figure 1 shows one iteration of the reverse helix, which can be repeated as many times as desired along the $z$-axis. This iteration is composed of two parts: the lower helix (here we define its rotation as clockwise) and the upper helix (we define its rotation as counter-clockwise), with rotation reversal at the kink point, $K$. For convenience, we denote the horizontal plane that goes through the kink point as kink plane. Without loss of generality, we assume that the kink plane is the plane $z=0$.

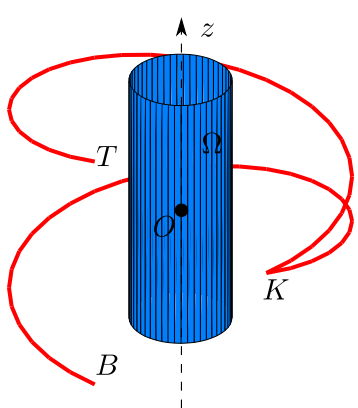

(a)

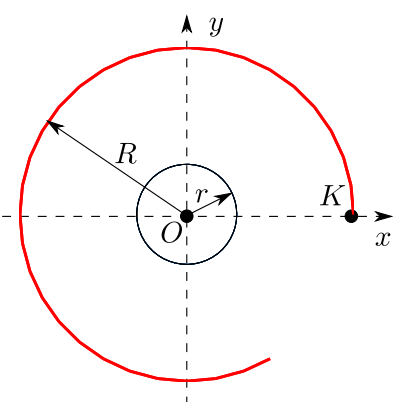

(b)
Fig. 1: Geometry of the reverse helical trajectory. (a) 3D-view; $\Omega$ is the region of interest. (b) Projection in the $(x, y)$-plane; $r$ : radius of the region of interest; $R$ : scan radius.

Let $R$ be the rotation radius and let $P$ be the pitch for each helix turning forming the reverse helix (this pitch is the distance traveled along the $z$-axis per $360^{\circ}$ ). Let $\underline{a}(\lambda)$ be the source position on the reverse helix, where $\lambda$ is the rotation angle. Without loss of generality, we define the rotation angle for the kink point as 0 , and the angular range for the lower helix as $\left[-\lambda_{m}, 0\right]$, whereas for the upper helix it is $\left[0, \lambda_{m}\right]$. Then the source position can be expressed as

$$
\begin{aligned}
& \underline{a}(\lambda)=
\end{aligned}
$$

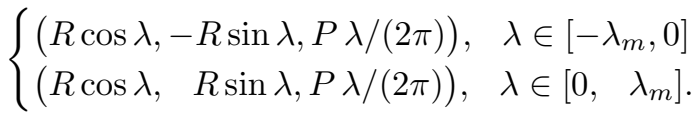

\section{B. Detector arrangement}

We consider a flat-panel detector that moves together with the vertex point, $\underline{a}(\lambda)$. The detector is at fixed distance $D$ from the source, and it is placed such that it is parallel to the $z$-axis and it is orthogonal to the plane defined by $\underline{a}(\lambda)$ and the $z$ axis; see Figure 2. For the representation of any point in the detector plane, we use a right-handed 3D Cartesian coordinate system defined by the following three orthogonal unit vectors:

$$
\left\{\begin{array}{l}
\underline{e}_{w}(\lambda)=(\cos \lambda, \sin |\lambda|, 0) \\
\underline{e}_{u}(\lambda)=(-\sin |\lambda|, \cos \lambda, 0) \\
\underline{e}_{v}(\lambda)=(0,0,1) .
\end{array}\right.
$$

We denote the axes that correspond to $\underline{e}_{u}(\lambda), \underline{e}_{v}(\lambda)$ and $\underline{e}_{w}(\lambda)$ as $u, v$ and $w$. The origin of this $3 \mathrm{D}$ coordinate system, i.e., $(u, v, w)=(0,0,0)$, corresponds to the orthogonal projection of the source point onto the detector. Note that $\underline{e}_{v}(\lambda)$ is parallel to the $z$-axis and $\underline{e}_{w}(\lambda)$ is pointing from the center of the detector to the source point.

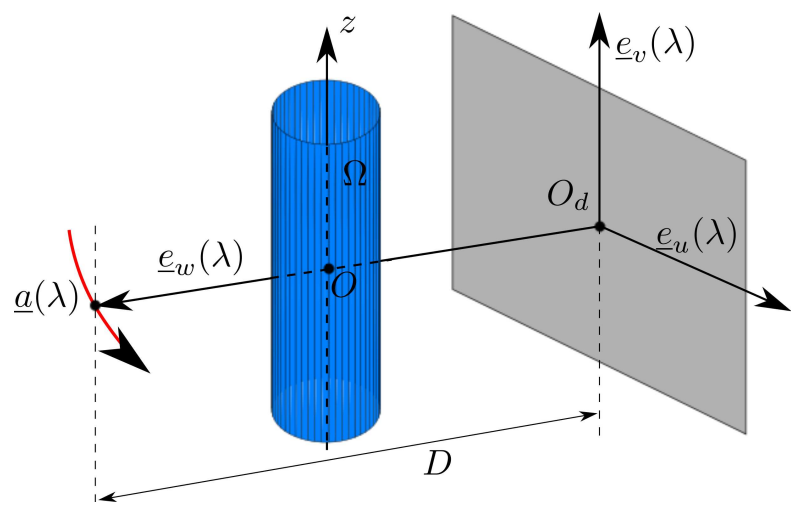

Fig. 2: Scan geometry and the detector arrangement.

Using the above notation, the CB projection from $\underline{a}(\lambda)$ to a point of detector coordinate $(u, v)$, can be expressed as:

$$
g(\lambda,, u, v)=\int_{0}^{\infty} f(\underline{a}(\lambda)+t \underline{\alpha}(\lambda, u, v)) d t
$$

where

$$
\underline{\alpha}(\lambda, u, v)=\frac{u \underline{e}_{u}(\lambda)+v \underline{e}_{v}(\lambda)-D \underline{e}_{w}(\lambda)}{\sqrt{u^{2}+v^{2}+D^{2}}} .
$$

\section{The spatial distribution of $\pi$-lines}

Note that Tuy's condition [7] is satisfied everywhere within the convex hull of the reverse helix. Hence, TES reconstruction from non-truncated projections is possible over the portion of $\Omega$ that is bounded by the convex hull of the reverse helix [8]. This data sufficiency condition and the currently available $\pi$-line based algorithms motivate the third method, VoxelDependent-HFDK, whose weighting scheme is designed from the spatial distribution of $\pi$-lines.

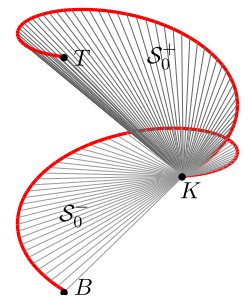

(a)

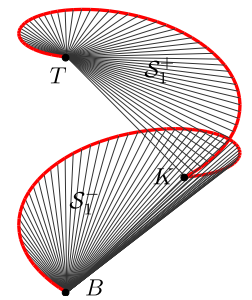

(b)

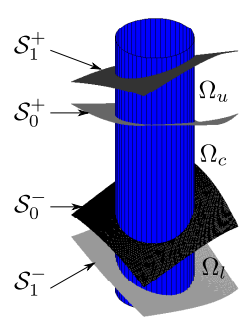

(c)
Fig. 3: The spatial distribution of $\pi$-lines of the reverse helix. (a) The $\pi$-line surfaces corresponding to the kink point $K$. (b) The $\pi$-line surfaces corresponding to the top and bottom points $T$ and $B$; (c) The spatial distribution of $\pi$-lines using the $\pi$-line surfaces. Spaces denoted by $\Omega_{u}$ and $\Omega_{l}$ are covered by $\pi$-lines, whereas $\Omega_{c}$ is not.

In Figure 3, we illustrate the spatial distribution of $\pi$-lines. Figure 3(a) and (b) show four $\pi$-surfaces: the two surfaces 
in (a) are generated by connecting the kink point $K$ to the points on the upper helix and to the points on the lower helix, respectively, whereas the two surfaces in (b) are obtained by connecting the endpoint $T$ (resp. $B$ ) to the other points of the upper (resp. lower) helix. The ROI $\Omega$ within the convex hull of the reverse helix can be divided into three parts: $\Omega_{u}$ and $\Omega_{l}$ covered by $\pi$-lines, and $\Omega_{c}$ not covered by $\pi$-lines, as depicted in Figure 3(c). In Voxel-Dependent-HFDK, we apply different weighting schemes depending on whether the point of interest belongs to $\Omega_{c}$ or not.

\section{Reconstruction Methods}

This section is devoted to explaining the three proposed FDK-type reconstruction methods for the reverse helix, which are extended from two fundamental FDK-type [9] formulae, one is ramp kernel based and the other is Hilbert kernel based. For convenience, we denote them as RFDK and HFDK, separately. In this work, all filtering lines are horizontal, i.e., ramp or Hilbert kernel based convolution is along lines that are parallel to the detector axis $\underline{e}_{u}(\lambda)$.

\section{A. Fusion-RFDK}

The Fusion-RFDK method consists of two stages. First, by RFDK, $\mathrm{Vol}^{+}$and $\mathrm{Vol}^{-}$are respectively reconstructed using data from the upper and lower helices, as shown in the left of Figure 4. We denote reconstructions in $\mathrm{Vol}^{+}$and $\mathrm{Vol}^{-}$as $f_{R}^{+}(\underline{x})$ and $f_{R}^{-}(\underline{x})$. Those two volumes are symmetric relative to the kink plane along with a fusion-zone, which is the overlapped region of $f_{R}^{+}(\underline{x})$ and $f_{R}^{-}(\underline{x})$. Let $[-H, H]$ be the $z$-range of the volume to be reconstructed, and let $L_{F}$ be the length of the fusion-zone. This indicates that the $z$-range of $\mathrm{Vol}^{+}$and $\mathrm{Vol}^{-}$are $\left[-0.5 L_{F}, H\right]$ and $\left[-H, 0.5 L_{F}\right]$.

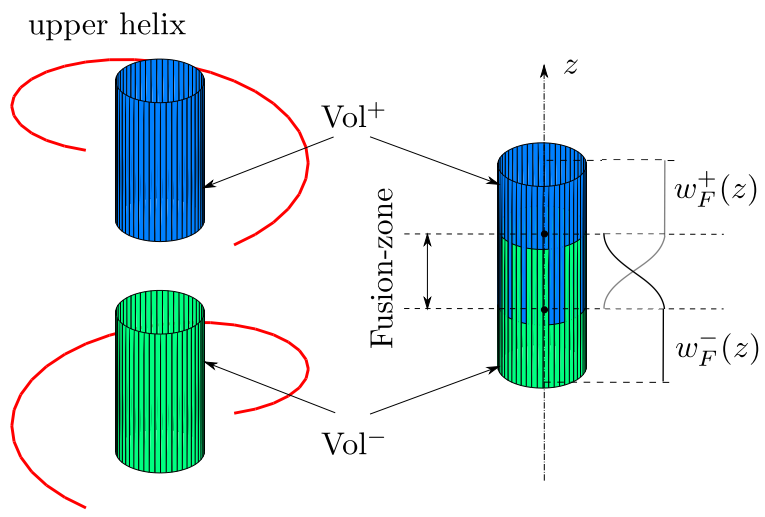

lower helix

Fig. 4: Illustration of the Fusion-RFDK reconstruction method. $\mathrm{Vol}^{+}$and $\mathrm{Vol}^{-}$are reconstructions from the upper and lower helices using RFDK. $w_{F}^{+}(z)$ and $w_{F}^{-}(z)$ are the fusion weighting functions for $\mathrm{Vol}^{+}$and $\mathrm{Vol}^{-} . L_{F}$ is the length of the fusion zone, within which feathering is applied.

Second, to obtain the final reconstruction result denoted $f_{F R}(\underline{x})$, we combine $f_{R}^{+}(\underline{x})$ and $f_{R}^{-}(\underline{x})$ by applying a feathering process to the horizontal slices covering the fusion-zone. This feathering process aims to smoothly incorporate both information from the upper and lower helices. The feathering process can be achieved by the use of a pair of fusion weighting functions $w_{F}^{+}(z)$ and $w_{F}^{-}(z)$, as shown in the right of Figure 4. Assigning the weights $w_{F}^{+}(z)$ and $w_{F}^{-}(z)$ to $f_{R}^{+}(\underline{x})$ and $f_{R}^{-}(\underline{x})$, we get two weighted volumes, the summation of which yields the final reconstruction of Fusion-RFDK. The exact expression for the two weights is

$$
\left\{\begin{array}{l}
w_{F}^{+}(z)= \begin{cases}1 & z \in\left[0.5 L_{F}, H\right] \\
\sin ^{2}\left(\frac{\pi\left(z+0.5 L_{F}\right)}{2 L_{F}}\right) & z \in\left[-0.5 L_{F}, 0.5 L_{F}\right) \\
0 & \text { otherwise }\end{cases} \\
w_{F}^{-}(z)= \begin{cases}1 & z \in\left[-H,-0.5 L_{F}\right] \\
\cos ^{2}\left(\frac{\pi\left(z+0.5 L_{F}\right)}{2 L_{F}}\right) & z \in\left(-0.5 L_{F}, 0.5 L_{F}\right] \\
0 & \text { otherwise. }\end{cases}
\end{array}\right.
$$

Let $w_{P}(\lambda, u)$ denote the Parker weighting [10] and let $h_{F}(u)$ be the ramp filter kernel, namely

$$
h_{F}(u)=\int_{\mathbb{R}}|\sigma| e^{i 2 \pi \sigma u} d \sigma .
$$

Also, let $u^{*}$ and $v^{*}$ be the coordinates for the projection of $\underline{x}$ onto the detector from $\underline{a}(\lambda)$, i.e.,

$$
\left\{\begin{array}{l}
u^{*}=\frac{D(\underline{x}-\underline{a}(\lambda)) \cdot \underline{e}_{u}(\lambda)}{R-\underline{x} \cdot \underline{e}_{w}(\lambda)}, \\
v^{*}=\frac{D(\underline{x}-\underline{a}(\lambda)) \cdot \underline{e}_{v}(\lambda)}{R-\underline{x} \cdot \underline{e}_{w}(\lambda)} .
\end{array}\right.
$$

Then, the reconstruction result for Fusion-RFDK, which we denote as $f_{F R}(\underline{x})$, can be obtained using the steps described below:

Step 1: CB length correction and Parker weighting:

$$
g_{1}(\lambda, u, v)=\frac{D}{\sqrt{u^{2}+v^{2}+D^{2}}} w_{P}(\lambda, u) g(\lambda, u, v) ;
$$

Step 2: ramp filtering along the $u$-axis:

$$
g_{R}(\lambda, u, v)=\int_{-\infty}^{\infty} d u^{\prime} h_{F}\left(u-u^{\prime}\right) g_{1}\left(\lambda, u^{\prime}, v\right) ;
$$

Step 3: backprojection:

$$
\begin{aligned}
& f_{R}^{+}(\underline{x})=\int_{0}^{\lambda_{m}} d \lambda \frac{R D}{\left(R-\underline{x} \cdot \underline{e}_{w}(\lambda)\right)^{2}} g_{R}\left(\lambda, u^{*}, v^{*}\right), \\
& f_{R}^{-}(\underline{x})=\int_{-\lambda_{m}}^{0} d \lambda \frac{R D}{\left(R-\underline{x} \cdot \underline{e}_{w}(\lambda)\right)^{2}} g_{R}\left(\lambda, u^{*}, v^{*}\right) ;
\end{aligned}
$$

Step 4: fusion:

$$
f_{F R}(\underline{x})=f_{R}^{+}(\underline{x}) w_{F}^{+}(z)+f_{R}^{-}(\underline{x}) w_{F}^{-}(z) .
$$

\section{B. Fusion-HFDK}

Fusion-HFDK shares the same fusion concept as that of Fusion-RFDK, the only difference is the algorithm used for obtaining $\mathrm{Vol}^{+}$and $\mathrm{Vol}^{-}$. Instead of using a ramp kernel based FDK-type formula, in Fusion-HFDK, a Hilbert kernel based formula is adopted. The reconstructed volumes, i.e., $\mathrm{Vol}^{+}$and $\mathrm{Vol}^{-}$, are denoted as $f_{H}^{+}(\underline{x})$ and $f_{H}^{-}(\underline{x})$ 
Let $h_{H}(u)=1 /(\pi u)$ denote the Hilbert filter kernel, and let $f_{F H}(\underline{x})$ be the final result of Fusion-HFDK, the implementation steps of Fusion-HFDK can be described as below.

Step 1: CB length correction and view-dependent differentiation

$$
g_{2}(\lambda, u, v)=\frac{D}{\sqrt{u^{2}+v^{2}+D^{2}}} g^{\prime}(\lambda, \underline{\alpha}(\lambda, u, v)) ;
$$

Step 2: Hilbert filtering along the $u$-axis

$$
g_{H}(\lambda, u, v)=\int_{-\infty}^{\infty} d u^{\prime} h_{H}\left(u-u^{\prime}\right) g_{2}\left(\lambda, u^{\prime}, v\right) ;
$$

Step 3: Parker weighting

$$
g_{P}(\lambda, u, v)=w_{P}(\lambda, u) g_{H}(\lambda, u, v) ;
$$

Step 4: backprojection

$$
\begin{aligned}
& f_{H}^{+}(\underline{x})=\frac{1}{2 \pi} \int_{0}^{\lambda_{m}} d \lambda g_{P}\left(\lambda, u^{*}, v^{*}\right), \\
& f_{H}^{-}(\underline{x})=\frac{1}{2 \pi} \int_{-\lambda_{m}}^{0} d \lambda g_{P}\left(\lambda, u^{*}, v^{*}\right) ;
\end{aligned}
$$

Step 5: fusion

$$
f_{F H}(\underline{x})=f_{H}^{+}(\underline{x}) w_{F}^{+}(z)+f_{H}^{-}(\underline{x}) w_{F}^{-}(z) .
$$

\section{Voxel-Dependent-HFDK}

The third method is called Voxel-Dependent-HFDK(VDHFDK), because the angular range of the backprojection and the weighting schemes are voxel dependent. The reconstruction using this method can be achieved by the following steps.

Step 1: CB length correction and view-dependent differentiation

$$
g_{2}(\lambda, u, v)=\frac{D}{\sqrt{u^{2}+v^{2}+D^{2}}} g^{\prime}(\lambda, \underline{\alpha}(\lambda, u, v)) ;
$$

Step 2: Hilbert filtering along the $u$-axis

$$
g_{H}(\lambda, u, v)=\int_{-\infty}^{\infty} d u^{\prime} h_{H}\left(u-u^{\prime}\right) g_{2}\left(\lambda, u^{\prime}, v\right)
$$

Step 3: voxel-dependent weighting

$$
g_{w}\left(\lambda, u^{*}, v^{*}\right)=w(\lambda, \underline{x}) g_{H}\left(\lambda, u^{*}, v^{*}\right) ;
$$

Step 4: voxel-dependent backprojection

$$
f(\underline{x})=\frac{1}{2 \pi} \int_{\Lambda(\underline{x})} d \lambda g_{w}^{\prime}\left(\lambda, u^{*}, v^{*}\right) .
$$

Note that the above steps are very similar to the first four steps of Fusion-HFDK, but with a different weighting scheme in step 3 and a different backprojection angular range in step 4 , denoted as $w(\lambda, \underline{x})$ and $\Lambda(\underline{x})$, respectively. Also note that, $w(\lambda, \underline{x})$ and $\Lambda(\underline{x})$ are voxel-dependent functions (depend on $\underline{x}$ ), and are designed differently according to the spatial distribution of $\pi$-lines, i.e., $\Omega_{u}, \Omega_{l}$ and $\Omega_{c}$, see Figure 3 . Details are given below.

1) $\underline{x} \in \Omega_{u}$ or $\underline{x} \in \Omega_{l}$ : As shown in Figure 5(a), the $\bar{\pi}$-segment is utilized for backprojection and a uniform

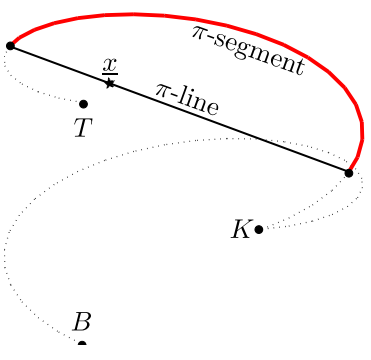

(a)

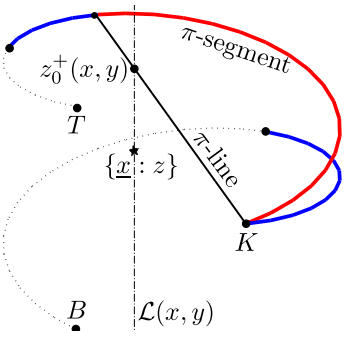

(b)
Fig. 5: Backprojection angular ranges for the third method: Voxel-Dependet-HFDK. (a) The point of interest $\underline{x}$ (black star) belongs to a $\pi$-line: $\pi$-segment (solid red) is used for backprojection. (b) Point $\underline{x}$ belongs to $\Omega_{c}$ : the backprojection angular range is designed by extending the corresponding $\pi$ segment (solid red) to its both ends (solid blue).

weight of 1 is assigned to projections that is within the $\pi$-segment.

2) $\underline{x} \in \Omega_{c}:$ As shown in Figure 5(b), the backprojection angular range and weighting scheme in this case is more complicated. Here we assume that the point of interest $\underline{x}$ is above the kink plane and below the $\pi$-surface $\mathcal{S}_{0}^{+}$. A similar explanation holds when $\underline{x}$ is below the kink plane and above the $\pi$-surface $\mathcal{S}_{0}^{-}$.

We denote the line that goes through $\underline{x}$ and is parallel to the $z$-axis as $\mathcal{L}(x, y)$. Together with the kink point $K$, this line defines a plane that intersects with the $\pi$-surface $\mathcal{S}_{0}^{+}$along a $\pi$-line, as shown by the solid black line. The angular range of the backprojection is designed by extending the $\pi$-segment, i.e., the solid red curve in Figure 5(b) at both ends (the extensions are indicated by the solid blue curves). The design criteria is such that the closer $\underline{x}$ is to $\mathcal{S}_{0}^{+}$, the smaller extensions are. In the extreme cases: i) when $\underline{x}$ is on the $\pi$ line, the extensions (solid blue) become nil; ii) when $\underline{x}$ is on the the kink plane, the whole reverse helix is used for the backprojection. Finally, a modified Parker weighting, $w(\lambda, \underline{x})$, is applied to the projections within the backprojection angular range $\Lambda(\underline{x})$; this weighting is based on a smooth function $c(\lambda)$ following the approach explained in [11].

\section{EVALUATION}

All the proposed methods either require horizontal ramp filtering or horizontal Hilbert transform, which could yield a lot of difference in terms of resolution. To perform a fair comparative evaluation, we have first adjusted all reconstruction parameters such that the three methods produce results with similar resolutions. Hence, all reconstructions shown here correspond to resolution-matched algorithms.

The evaluations were based on computer-simulated data of the FORBILD head phantom, shown in Figure 6. Two angular ranges were used for the reverse helix, i.e., $240^{\circ}$ and $360^{\circ}$. In each case, the height of the reverse helix (distance between $B$ and $T$ in Figure 1) and the number of views for 


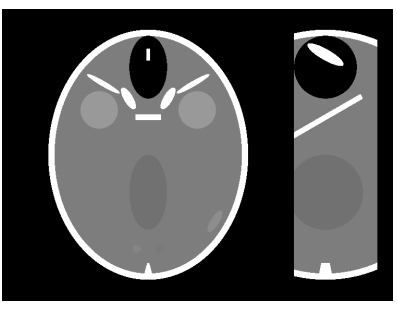

Fig. 6: FORBILD head phantom (gray scale: [0 100]HU). Left: cross section in the $(x, y)$-plane. Right: cross section in the $(y, z)$-plane with $z \in[-3 \mathrm{~cm}, 5 \mathrm{~cm}]$.

each helix turning were fixed to $12 \mathrm{~cm}$ and 600 , respectively. Also, the kink plane was positioned one centimeter above the central plane through the phantom, to break symmetry between the phantom and the source trajectory. The radius $R$ and the source-to-detector distance $D$ used for evaluations were $78.5 \mathrm{~cm}$ and $119.9 \mathrm{~cm}$, respectively. Projection data were measured by the detector that consisted of $700 \times 700$ square bins of side $0.0616 \mathrm{~cm}$.

\section{A. Fusion effects}

A fusion zone of $z \in[-1 \mathrm{~cm}, 3 \mathrm{~cm}]$ was used for FusionRFDK and Fusion-HFDK. As shown in Figure 7, the fusion process has a significant impact on cone-beam artifacts: the strong $\mathrm{CB}$ artifacts around the bone areas in the left and right columns have nearly opposite intensities, and thus are considerably reduced by the fusion process. Figure 7 only shows results for angular range of $240^{\circ}$, but the same observations holds for the $360^{\circ}$ range.
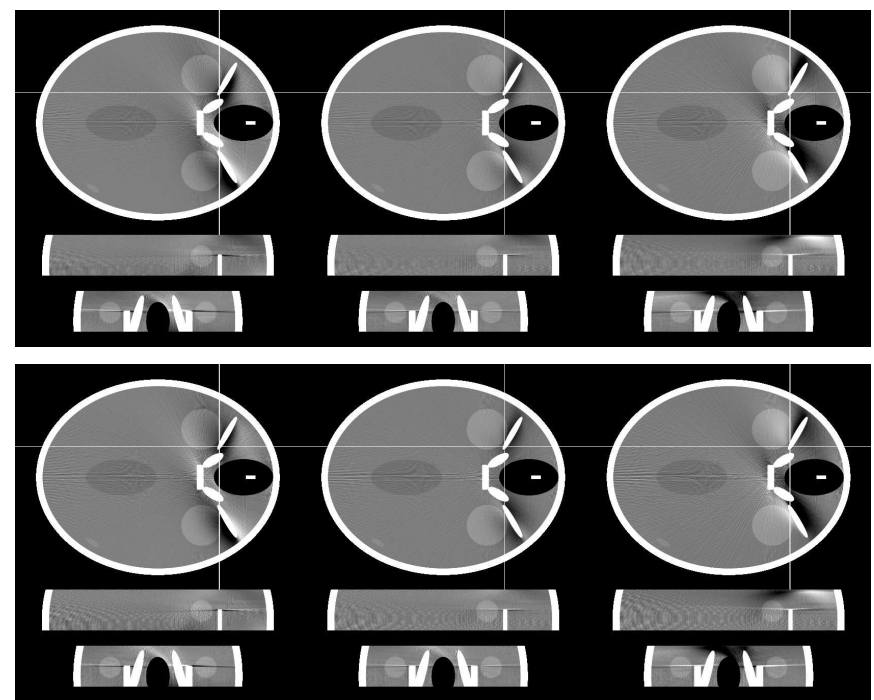

Fig. 7: Fusion effects for scanning angular range of $240^{\circ}$ (gray scale: [0 100]HU), the horizontal cross section is in the plane $z=-0.05 \mathrm{~cm}$. Upper: Fusion-RFDK; lower: Fusion-HFDK. First column: reconstruction results by only using projection data from the upper helix; second column: reconstruction result after fusion process; third column: reconstruction results by only using projection data from the lower helix.

\section{B. Visual inspection}

Figure 8 shows the reconstruction results from all three methods using angular ranges of $240^{\circ}$ and $360^{\circ}$. For $240^{\circ}$, less CB artifacts were observed around the frontal sinus (upper black ellipsoid with high contrast) in the results from the Fusion-HFDK and VD-HFDK than that from the FusionRFDK. However, regarding CB artifacts around the ventricle (middle gray ellipsoid with low contrast), Fusion-RFDK outperforms the other two methods. Different behavior of CB artifacts can be observed for $360^{\circ}$. Fusion-RFDK produces more $\mathrm{CB}$ artifacts around both the frontal sinus and ventricle than Fusion-HFDK or VD-HFDK. The observations indicate, not surprisingly, that the $\mathrm{CB}$ artifacts of all three methods are location, object and angular range dependent. Overall, all three methods produce images of acceptable quality, and no artifacts are observed related to the transition from the core to the region outside of the core.

As expected, in the $\pi$-line regions (e.g., area close to the left edge of the cross section in the $(y, z)$ plane), VD-HFDK produces little $\mathrm{CB}$ artifacts and outperforms the other two methods. However, the $\pi$-line regions only occupy a relatively small portion of the whole reconstruction volume.
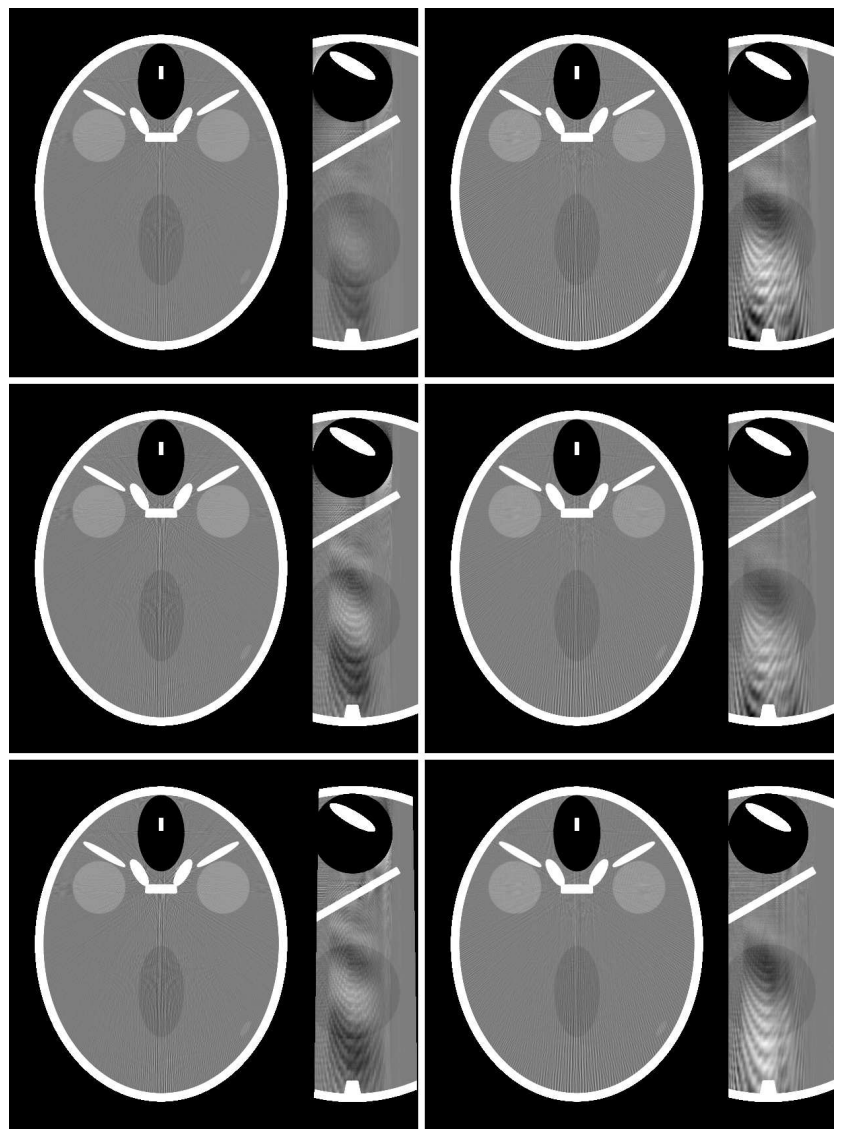

Fig. 8: Reconstruction results (gray scale: [0,100]HU). First column: $240^{\circ}$; second column: $360^{\circ}$. First row: Fusion-RFDK; second row: Fusion-HFDK; third row: VD-HFDK. 


\section{CONCLUSIONS}

We have proposed three FDK-type reconstruction methods for the reverse helix, they are simple, stable and thus practical. The computer-simulation results show that all three methods produce acceptable images, and the images are smooth near the boundary of the core. Also, they only require one backprojection and no forward projection, thus they are more efficient compared to the previous solutions. We found that the $\mathrm{CB}$ artifacts are location, object and angular range dependent. For Fusion-RFDK and Fusion-HFDK, we found that the artifacts are strongly mitigated through the fusion process.

Although VD-HFDK can provide almost TES results in the $\pi$-regions, we found it to be only moderately attractive over Fusion-RFDK and Fusion-HFDK because the $\pi$-region, due to its size being relatively small, turns out to have little impact on the overall image quality, whereas the associated reconstruction steps are much more complicated. Determining which of the first two algorithms is preferable in terms of image quality is not clear based on the results we presented and will require further investigation. As far as computational effort is concerned, these two algorithms are very similar and can be easily implemented within the currently-used industrial reconstruction framework.

\section{REFERENCES}

[1] S. Cho, D. Xia, P. C. A., and X. Pan, "Exact reconstruction of volumetric images in reverse helical cone-beam ct," Med. Phys, vol. 35, pp. 303040, 2008.

[2] F. Noo, A. Wunderlich, G. Lauritsch, and H. Kudo, "On the problem of axial data truncation in the reverse helix geometry," in 10th International Meeting on Fully Three-Dimensional Image Reconstruction in Radiology and Nuclear Medicine, Beijing, China, 2009.

[3] S. Cho, D. Xia, P. C. A., and X. Pan, "A bpf-fbp tandem algorithm for image reconstrution in reverse helical cone-beam ct," Med. Phys, vol. 37, pp. 32-39, 2010.

[4] J. D. Pack and F. Noo, "Cone-beam reconstruction outside r-lines using the backprojection of 1-d filtered data," in 8th International Meeting on Fully Three-Dimensional Image Reconstruction in Radiology and Nuclear Medicine, Salt Lake City, U.S.A., 2005.

[5] H. Turbell and P. Danielsson, "An improved PI-method for reconstruction from helical cone-beam projections," in IEEE nuclear science and medical imaging symposium, Seattle, WA, USA, 1999.

[6] K. Sourbelle and W. Kalender, "Generalization of Feldkamp reconstruction for clinical spiral cone-beam CT," in The 7th International Meeting on Fully Three-Dimensional Image Reconstruction in Radiology and Nuclear Medicine, Saint Malo, France, 2003.

[7] H. Tuy, "An inversion formula for cone-beam reconstruction," SIAM J. Appl. Math, vol. 43, no. 3, pp. 546-552, June 1983.

[8] D. Finch, "Cone-beam reconstruction with sources on a curve," SIAM J. Appl. Math, vol. 45, no. 4, pp. 665-673, August 1985.

[9] L. A. Feldkamp, L. C. Davis, and J. W. Kress, "Practical cone-beam algorithm," J. Opt. Soc. Amer. A, vol. 1, no. 6, pp. 612-619, June 1984.

[10] D. Parker, "Optimal short scan convolution reconstruction for fan-beam CT," Med. Phys., vol. 9, pp. 254-7, 1982.

[11] F. Noo, M. Defrise, R. Clackdoyle, and H. Kudo, "Image reconstruction from fan-beam projections on less than a short scan," Phys.Med.Biol, vol. 47, pp. 2525-46, July 2002. 